\title{
SUPPOTING INFORMATION
}

\section{The first mixed-anion complex of a lithium phosphazene:}

\author{
synthesis, crystal and solution structure of \\ $\left[\left(\mathrm{LiCH}_{2} \mathrm{P}(\mathrm{Ph})_{2}=\mathrm{NPh}\right) \cdot\left(\mathrm{LiOC}_{6} \mathrm{H}_{2}-2,6-\left\{\mathrm{C}\left(\mathrm{CH}_{3}\right)_{3}\right\}-4-\mathrm{CH}_{3}\right)\right]_{2}$
}

Richard D. Price, ${ }^{a}$ Ignacio Fernández, ${ }^{b}$ Gloria Ruiz Gómez, ${ }^{b}$ Fernando López Ortiz, ${ }^{* b}$ Matthew G. Davidson, ${ }^{* a}$ John A. Cowan, ${ }^{c}$ and Judith A. K. Howard ${ }^{c}$

${ }^{a}$ Department of Chemistry, Claverton Down, University of Bath, Bath, BA2 7AY, UK.

b Área de Química Orgánica, Universidad de Almería, Carretera de Sacramento, 04120, Almería, Spain.

${ }^{c}$ Department of Chemistry, University of Durham, South Road Durham DH1 3LE UK.

\section{Contents:}

- X-Ray data of compound 10 (Figures S1 and S2, Tables S1-S5).

- Figure S3. 1D gROESY spectra observed of $\mathbf{1 0}$ in toluene- $d_{8}$ at $-70^{\circ} \mathrm{C}$.

- Figure $\mathbf{S 4} .2 \mathrm{D}{ }^{7} \mathrm{Li},{ }^{31} \mathrm{P}\left\{{ }^{1} \mathrm{H}\right\} \mathrm{HMQC}$ spectrum of complex 10 at $-80{ }^{\circ} \mathrm{C}$.

- Figure $55{ }^{13} \mathrm{C}\left\{{ }^{1} \mathrm{H}\right\}$ and ${ }^{13} \mathrm{C}\left\{{ }^{1} \mathrm{H},{ }^{31} \mathrm{P}\right\}$ NMR spectrum (125.75 MHz) of $\mathbf{1 0}$ measured at $-70{ }^{\circ} \mathrm{C}$ in toluene- $d_{8}$. 


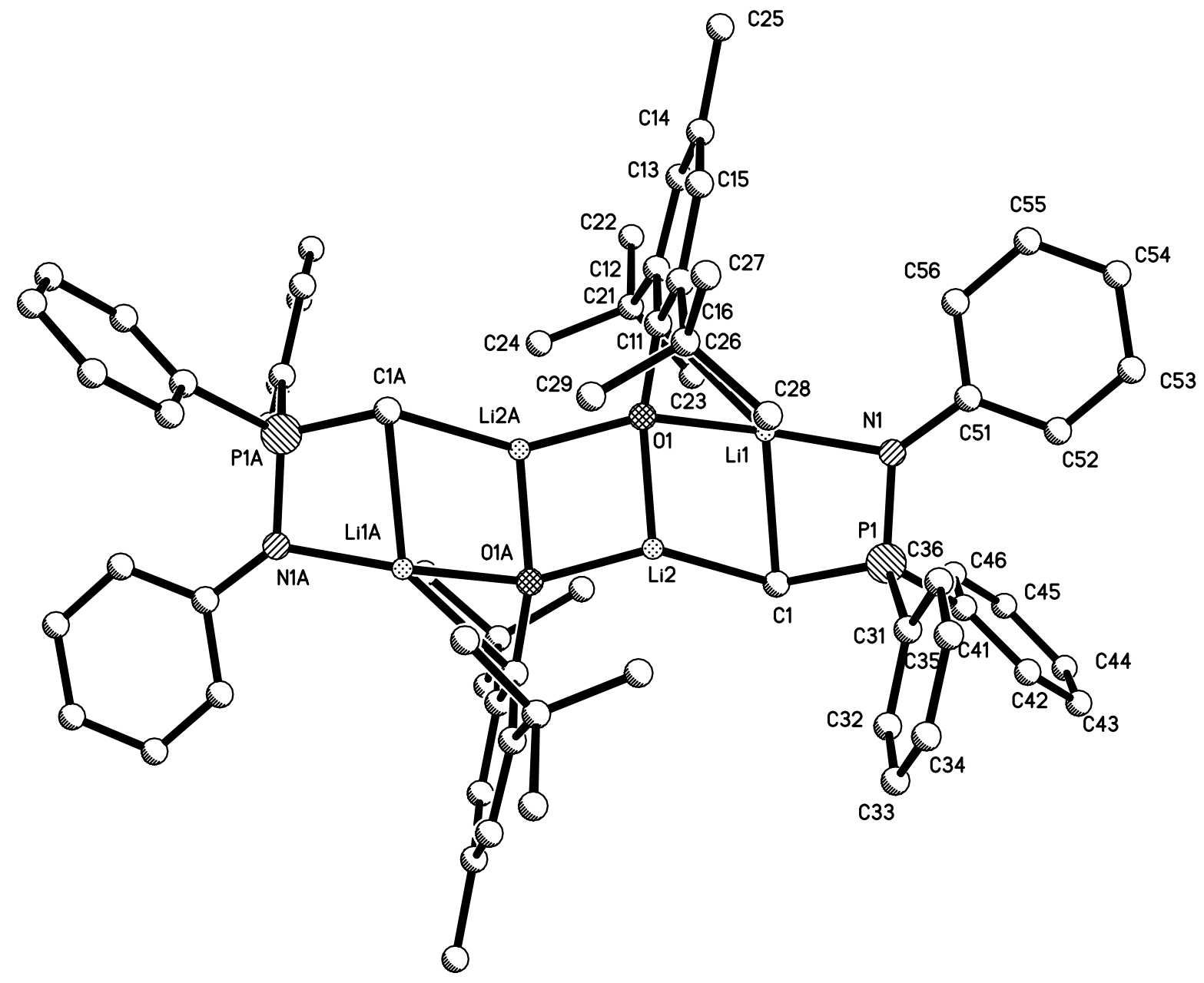

Fig. S1: Numbering Scheme for compound 10
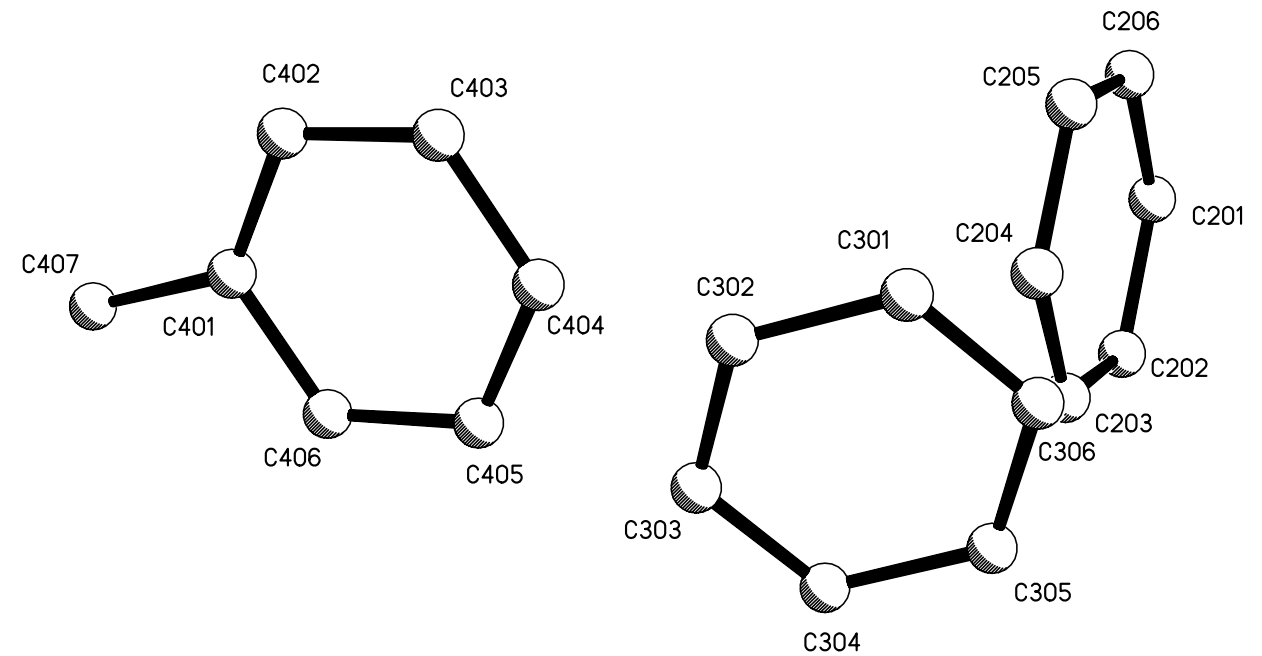

Fig. S2: Numbering Scheme for lattice solvent in the structure of compound $\mathbf{1 0}$ 
Table S1. Crystal data and structure refinement for Compound $\mathbf{1 0 .}$

Identification code

Empirical formula

Formula weight

Temperature

Wavelength

Crystal system

Space group

Unit cell dimensions

Volume

Z

Density (calculated)

Absorption coefficient

$\mathrm{F}(000)$

Crystal size

Theta range for data collection

Index ranges

Reflections collected

Independent reflections

Completeness to theta $=27.40^{\circ}$

Absorption correction

Refinement method

Data / restraints / parameters

Goodness-of-fit on $\mathrm{F}^{2}$

Final $R$ indices [I $>2 \operatorname{sigma}(\mathrm{I})]$

$\mathrm{R}$ indices (all data)

Largest diff. peak and hole jac $3 b$

C84.17 H96.77 Li4 N2 O2 P2

1258.17

100(2) K

$0.71073 \AA$

Monoclinic

$\mathrm{C} 2 / \mathrm{c}$

$\mathrm{a}=25.007(6) \AA$

$\alpha=90^{\circ}$.

$\mathrm{b}=13.561(3) \AA$

$\beta=107.43(5)^{\circ}$.

$c=24.345(5) \AA$ $\gamma=90^{\circ}$.

7877(3) $\AA^{3}$

4

$1.061 \mathrm{Mg} / \mathrm{m}^{3}$

$0.100 \mathrm{~mm}^{-1}$

2695.2

$0.2 \times 0.11 \times 0.1 \mathrm{~mm}^{3}$

3.31 to $27.40^{\circ}$.

$-30<=\mathrm{h}<=30,-17<=\mathrm{k}<=11,-31<=\mathrm{l}<=29$

28193

$7740[\mathrm{R}(\mathrm{int})=0.0720]$

$86.3 \%$

None

Full-matrix least-squares on $\mathrm{F}^{2}$

7740 / 0 / 493

1.206

$\mathrm{R} 1=0.1029, \mathrm{wR} 2=0.2374$

$\mathrm{R} 1=0.1195, \mathrm{wR} 2=0.2476$

0.775 and -0.583 e. $\AA^{-3}$ 
Table S2. Atomic coordinates ( $\left.\mathrm{x} 10^{4}\right)$ and equivalent isotropic displacement parameters $\left(\AA^{2} \times 10^{3}\right)$ for Compound 10. $\mathrm{U}(\mathrm{eq})$ is defined as one third of the trace of the orthogonalized $\mathrm{U}^{\mathrm{ij}}$ tensor.

\begin{tabular}{|c|c|c|c|c|}
\hline & $\mathrm{x}$ & $\mathrm{y}$ & $\mathrm{z}$ & $\mathrm{U}(\mathrm{eq})$ \\
\hline $\mathrm{P}(1)$ & $2608(1)$ & $52(1)$ & $3711(1)$ & $34(1)$ \\
\hline $\mathrm{O}(1)$ & 1955(1) & $2359(2)$ & $4536(1)$ & $34(1)$ \\
\hline $\mathrm{N}(1)$ & $1945(2)$ & $407(2)$ & $3412(1)$ & $36(1)$ \\
\hline$C(1)$ & $2765(2)$ & $403(3)$ & $4432(2)$ & $41(1)$ \\
\hline $\mathrm{C}(11)$ & $1403(2)$ & 2618(3) & $4220(1)$ & $35(1)$ \\
\hline$C(12)$ & $916(2)$ & 2188(4) & $4338(2)$ & $55(1)$ \\
\hline$C(13)$ & $361(2)$ & $2408(4)$ & $3979(2)$ & $63(2)$ \\
\hline$C(14)$ & $264(2)$ & $3068(4)$ & $3527(2)$ & $47(1)$ \\
\hline$C(15)$ & $740(2)$ & $3509(3)$ & $3430(2)$ & $36(1)$ \\
\hline$C(16)$ & $1314(2)$ & $3303(3)$ & $3750(1)$ & $30(1)$ \\
\hline $\mathrm{C}(21)$ & $981(2)$ & $1486(5)$ & $4857(2)$ & $81(2)$ \\
\hline$C(22)$ & $391(3)$ & $1156(10)$ & $4905(3)$ & $190(7)$ \\
\hline$C(23)$ & $1305(4)$ & $543(5)$ & $4802(2)$ & $114(4)$ \\
\hline$C(24)$ & $1294(2)$ & $2034(5)$ & $5424(2)$ & $60(2)$ \\
\hline$C(25)$ & $-352(2)$ & $3327(5)$ & $3169(2)$ & $61(2)$ \\
\hline$C(26)$ & $1823(2)$ & 3791(3) & $3590(1)$ & $29(1)$ \\
\hline$C(27)$ & $1611(2)$ & $4548(3)$ & $3098(2)$ & $36(1)$ \\
\hline$C(28)$ & $2170(2)$ & $3008(3)$ & $3372(2)$ & $34(1)$ \\
\hline$C(29)$ & $2230(2)$ & $4349(3)$ & 4101(2) & $31(1)$ \\
\hline$C(31)$ & $3144(2)$ & $660(3)$ & $3437(2)$ & $40(1)$ \\
\hline$C(32)$ & $3735(2)$ & $572(4)$ & $3723(2)$ & $56(1)$ \\
\hline $\mathrm{C}(33)$ & $4138(3)$ & $1118(5)$ & $3549(2)$ & $67(2)$ \\
\hline$C(34)$ & $3954(3)$ & $1748(4)$ & $3082(2)$ & $68(2)$ \\
\hline$C(35)$ & $3370(3)$ & 1831(3) & 2791(2) & $58(2)$ \\
\hline$C(36)$ & $2959(2)$ & 1293(3) & $2963(2)$ & $45(1)$ \\
\hline $\mathrm{C}(41)$ & $2710(2)$ & $-1270(3)$ & $3622(2)$ & $36(1)$ \\
\hline $\mathrm{C}(42)$ & $3096(2)$ & $-1664(3)$ & $3363(2)$ & $36(1)$ \\
\hline $\mathrm{C}(43)$ & $3167(2)$ & $-2684(3)$ & $3334(2)$ & $43(1)$ \\
\hline $\mathrm{C}(44)$ & $2837(3)$ & $-3300(3)$ & $3563(2)$ & $60(2)$ \\
\hline$C(45)$ & 2444(3) & $-2918(3)$ & $3817(3)$ & $74(2)$ \\
\hline $\mathrm{C}(46)$ & $2376(3)$ & $-1901(3)$ & $3848(2)$ & $58(1)$ \\
\hline
\end{tabular}







Table S3. Bond lengths $[\AA]$ and angles $\left[{ }^{\circ}\right]$ for Compound $\mathbf{1 0}$.

\begin{tabular}{|c|c|c|c|}
\hline $\mathrm{P}(1)-\mathrm{N}(1)$ & $1.670(4)$ & $\mathrm{C}(23)-\mathrm{H}(23 \mathrm{~B})$ & 0.9773 \\
\hline $\mathrm{P}(1)-\mathrm{C}(1)$ & $1.747(4)$ & $\mathrm{C}(23)-\mathrm{H}(23 \mathrm{C})$ & 0.9795 \\
\hline $\mathrm{P}(1)-\mathrm{C}(41)$ & $1.831(4)$ & $\mathrm{C}(24)-\mathrm{H}(24 \mathrm{~A})$ & 0.9801 \\
\hline $\mathrm{P}(1)-\mathrm{C}(31)$ & $1.858(4)$ & $\mathrm{C}(24)-\mathrm{H}(24 \mathrm{~B})$ & 0.9796 \\
\hline $\mathrm{P}(1)-\operatorname{Li}(1)$ & $2.697(9)$ & $\mathrm{C}(24)-\mathrm{H}(24 \mathrm{C})$ & 0.9802 \\
\hline $\mathrm{O}(1)-\mathrm{C}(11)$ & $1.409(5)$ & $\mathrm{C}(25)-\mathrm{H}(25 \mathrm{~A})$ & 0.9800 \\
\hline $\mathrm{O}(1)-\operatorname{Li}(2) \# 1$ & $1.909(7)$ & $\mathrm{C}(25)-\mathrm{H}(25 \mathrm{~B})$ & 0.9800 \\
\hline $\mathrm{O}(1)-\mathrm{Li}(1)$ & $1.939(8)$ & $\mathrm{C}(25)-\mathrm{H}(25 \mathrm{C})$ & 0.9801 \\
\hline $\mathrm{O}(1)-\mathrm{Li}(2)$ & $1.988(9)$ & $C(26)-C(27)$ & $1.545(5)$ \\
\hline $\mathrm{N}(1)-\mathrm{C}(51)$ & $1.407(5)$ & $C(26)-C(29)$ & $1.549(5)$ \\
\hline $\mathrm{N}(1)-\operatorname{Li}(1)$ & $1.936(8)$ & $C(26)-C(28)$ & $1.559(5)$ \\
\hline$C(1)-\operatorname{Li}(2)$ & $2.164(8)$ & $\mathrm{C}(27)-\mathrm{H}(27 \mathrm{~A})$ & 0.9793 \\
\hline $\mathrm{C}(1)-\operatorname{Li}(1)$ & $2.372(10)$ & $\mathrm{C}(27)-\mathrm{H}(27 \mathrm{~B})$ & 0.9800 \\
\hline $\mathrm{C}(1)-\mathrm{H}(1 \mathrm{~A})$ & 1.0446 & $\mathrm{C}(27)-\mathrm{H}(27 \mathrm{C})$ & 0.9799 \\
\hline $\mathrm{C}(1)-\mathrm{H}(1 \mathrm{~B})$ & 1.0069 & $\mathrm{C}(28)-\mathrm{H}(28 \mathrm{~A})$ & 0.9785 \\
\hline$C(11)-C(16)$ & $1.438(5)$ & $\mathrm{C}(28)-\mathrm{H}(28 \mathrm{~B})$ & 0.9800 \\
\hline$C(11)-C(12)$ & $1.456(6)$ & $\mathrm{C}(28)-\mathrm{H}(28 \mathrm{C})$ & 0.9797 \\
\hline$C(11)-\operatorname{Li}(1)$ & $2.371(10)$ & $\mathrm{C}(29)-\mathrm{H}(29 \mathrm{~A})$ & 0.9790 \\
\hline$C(12)-C(13)$ & $1.432(7)$ & $\mathrm{C}(29)-\mathrm{H}(29 \mathrm{~B})$ & 0.9797 \\
\hline$C(12)-C(21)$ & $1.551(6)$ & $\mathrm{C}(29)-\mathrm{H}(29 \mathrm{C})$ & 0.9795 \\
\hline$C(13)-C(14)$ & $1.382(6)$ & $\mathrm{C}(31)-\mathrm{C}(36)$ & $1.402(6)$ \\
\hline $\mathrm{C}(13)-\mathrm{H}(13 \mathrm{~A})$ & 0.9495 & $C(31)-C(32)$ & $1.437(7)$ \\
\hline$C(14)-C(15)$ & $1.413(6)$ & $\mathrm{C}(32)-\mathrm{C}(33)$ & $1.414(7)$ \\
\hline$C(14)-C(25)$ & $1.566(7)$ & $\mathrm{C}(32)-\mathrm{H}(32 \mathrm{~A})$ & 0.9500 \\
\hline$C(15)-C(16)$ & $1.441(6)$ & $\mathrm{C}(33)-\mathrm{C}(34)$ & $1.385(8)$ \\
\hline $\mathrm{C}(15)-\mathrm{H}(15 \mathrm{~A})$ & 0.9500 & $\mathrm{C}(33)-\mathrm{H}(33 \mathrm{~A})$ & 0.9498 \\
\hline$C(16)-C(26)$ & $1.585(5)$ & $\mathrm{C}(34)-\mathrm{C}(35)$ & $1.424(9)$ \\
\hline$C(21)-C(23)$ & $1.542(11)$ & $\mathrm{C}(34)-\mathrm{H}(34 \mathrm{~A})$ & 0.9503 \\
\hline$C(21)-C(24)$ & $1.557(7)$ & $\mathrm{C}(35)-\mathrm{C}(36)$ & $1.421(7)$ \\
\hline$C(21)-C(22)$ & $1.578(9)$ & $\mathrm{C}(35)-\mathrm{H}(35 \mathrm{~A})$ & 0.9500 \\
\hline $\mathrm{C}(22)-\mathrm{H}(22 \mathrm{~A})$ & 0.9799 & $\mathrm{C}(36)-\mathrm{H}(36 \mathrm{~A})$ & 0.9500 \\
\hline $\mathrm{C}(22)-\mathrm{H}(22 \mathrm{~B})$ & 0.9799 & $\mathrm{C}(41)-\mathrm{C}(42)$ & $1.408(6)$ \\
\hline $\mathrm{C}(22)-\mathrm{H}(22 \mathrm{C})$ & 0.9798 & $\mathrm{C}(41)-\mathrm{C}(46)$ & $1.417(6)$ \\
\hline $\mathrm{C}(23)-\mathrm{H}(23 \mathrm{~A})$ & 0.9811 & $\mathrm{C}(42)-\mathrm{C}(43)$ & $1.399(6)$ \\
\hline
\end{tabular}




\begin{tabular}{|c|c|c|c|}
\hline $\mathrm{C}(42)-\mathrm{H}(42 \mathrm{~A})$ & 0.9498 & $C(301)-C(306)$ & 1.3900 \\
\hline $\mathrm{C}(43)-\mathrm{C}(44)$ & $1.402(6)$ & $\mathrm{C}(301)-\mathrm{H}(301)$ & 0.9300 \\
\hline $\mathrm{C}(43)-\mathrm{H}(43 \mathrm{~A})$ & 0.9500 & $\mathrm{C}(302)-\mathrm{C}(303)$ & 1.3900 \\
\hline $\mathrm{C}(44)-\mathrm{C}(45)$ & $1.408(7)$ & $\mathrm{C}(302)-\mathrm{H}(302)$ & 0.9300 \\
\hline $\mathrm{C}(44)-\mathrm{H}(44 \mathrm{~A})$ & 0.9499 & $\mathrm{C}(303)-\mathrm{C}(304)$ & 1.3900 \\
\hline$C(45)-C(46)$ & $1.394(6)$ & $\mathrm{C}(303)-\mathrm{H}(303)$ & 0.9300 \\
\hline $\mathrm{C}(45)-\mathrm{H}(45 \mathrm{~A})$ & 0.9500 & $C(304)-C(305)$ & 1.3900 \\
\hline $\mathrm{C}(46)-\mathrm{H}(46 \mathrm{~A})$ & 0.9500 & $\mathrm{C}(304)-\mathrm{H}(304)$ & 0.9300 \\
\hline$C(51)-C(52)$ & $1.412(6)$ & $C(305)-C(306)$ & 1.3900 \\
\hline $\mathrm{C}(51)-\mathrm{C}(56)$ & $1.441(6)$ & $\mathrm{C}(305)-\mathrm{H}(305)$ & 0.9300 \\
\hline$C(52)-C(53)$ & $1.402(6)$ & $\mathrm{C}(306)-\mathrm{H}(306)$ & 0.9300 \\
\hline $\mathrm{C}(52)-\mathrm{H}(52 \mathrm{~A})$ & 0.9497 & $\mathrm{C}(401)-\mathrm{C}(405) \# 2$ & $0.38(3)$ \\
\hline $\mathrm{C}(53)-\mathrm{C}(54)$ & $1.422(7)$ & $\mathrm{C}(401)-\mathrm{C}(406) \# 2$ & $1.21(3)$ \\
\hline $\mathrm{C}(53)-\mathrm{H}(53 \mathrm{~A})$ & 0.9501 & $\mathrm{C}(401)-\mathrm{C}(404) \# 2$ & $1.331(7)$ \\
\hline $\mathrm{C}(54)-\mathrm{C}(55)$ & $1.396(7)$ & $C(401)-C(402)$ & 1.3900 \\
\hline $\mathrm{C}(54)-\mathrm{H}(54 \mathrm{~A})$ & 0.9503 & $\mathrm{C}(401)-\mathrm{C}(406)$ & 1.3900 \\
\hline$C(55)-C(56)$ & $1.393(6)$ & $C(401)-C(407)$ & $1.591(17)$ \\
\hline $\mathrm{C}(55)-\mathrm{H}(55 \mathrm{~A})$ & 0.9499 & $\mathrm{C}(402)-\mathrm{C}(403)$ & 1.3900 \\
\hline $\mathrm{C}(56)-\mathrm{H}(56 \mathrm{~A})$ & 0.9502 & $\mathrm{C}(402)-\mathrm{C}(402) \# 2$ & $1.95(2)$ \\
\hline $\operatorname{Li}(1)-\operatorname{Li}(2)$ & $2.499(10)$ & $\mathrm{C}(402)-\mathrm{H}(402)$ & 0.9300 \\
\hline $\mathrm{Li}(1)-\mathrm{H}(23 \mathrm{~A})$ & 2.0198 & $\mathrm{C}(403)-\mathrm{C}(404)$ & 1.3900 \\
\hline $\mathrm{Li}(2)-\mathrm{O}(1) \# 1$ & $1.909(7)$ & $\mathrm{C}(403)-\mathrm{H}(403)$ & 0.9300 \\
\hline $\operatorname{Li}(2)-\operatorname{Li}(2) \# 1$ & $2.490(16)$ & $\mathrm{C}(404)-\mathrm{C}(405)$ & 1.3900 \\
\hline$C(201)-C(202)$ & 1.3900 & $\mathrm{C}(404)-\mathrm{H}(404)$ & 0.9300 \\
\hline$C(201)-C(206)$ & 1.3900 & $\mathrm{C}(405)-\mathrm{C}(406)$ & 1.3900 \\
\hline $\mathrm{C}(201)-\mathrm{H}(201)$ & 0.9300 & $\mathrm{C}(405)-\mathrm{H}(405)$ & 0.9300 \\
\hline$C(202)-C(203)$ & 1.3900 & $\mathrm{C}(406)-\mathrm{H}(406)$ & 0.9300 \\
\hline $\mathrm{C}(202)-\mathrm{H}(202)$ & 0.9300 & $\mathrm{C}(407)-\mathrm{C}(405) \# 2$ & $1.26(2)$ \\
\hline C(203)-C(204) & 1.3900 & $\mathrm{C}(407)-\mathrm{H}(40 \mathrm{~F})$ & 0.9600 \\
\hline $\mathrm{C}(203)-\mathrm{H}(203)$ & 0.9300 & $\mathrm{C}(407)-\mathrm{H}(40 \mathrm{G})$ & 0.9600 \\
\hline$C(204)-C(205)$ & 1.3900 & $\mathrm{C}(407)-\mathrm{H}(40 \mathrm{H})$ & 0.9600 \\
\hline $\mathrm{C}(204)-\mathrm{H}(204)$ & 0.9300 & & \\
\hline$C(205)-C(206)$ & 1.3900 & $\mathrm{~N}(1)-\mathrm{P}(1)-\mathrm{C}(1)$ & $104.7(2)$ \\
\hline $\mathrm{C}(205)-\mathrm{H}(205)$ & 0.9300 & $\mathrm{~N}(1)-\mathrm{P}(1)-\mathrm{C}(41)$ & $112.66(18)$ \\
\hline $\mathrm{C}(206)-\mathrm{H}(206)$ & 0.9300 & $\mathrm{C}(1)-\mathrm{P}(1)-\mathrm{C}(41)$ & $112.84(19)$ \\
\hline $\mathrm{C}(301)-\mathrm{C}(302)$ & 1.3900 & $\mathrm{~N}(1)-\mathrm{P}(1)-\mathrm{C}(31)$ & $116.38(19)$ \\
\hline
\end{tabular}




\begin{tabular}{|c|c|c|c|}
\hline $\mathrm{C}(1)-\mathrm{P}(1)-\mathrm{C}(31)$ & $105.70(19)$ & $\mathrm{C}(12)-\mathrm{C}(13)-\mathrm{H}(13 \mathrm{~A})$ & 119.1 \\
\hline $\mathrm{C}(41)-\mathrm{P}(1)-\mathrm{C}(31)$ & $104.59(19)$ & $C(13)-C(14)-C(15)$ & $116.9(4)$ \\
\hline $\mathrm{N}(1)-\mathrm{P}(1)-\mathrm{Li}(1)$ & $45.50(19)$ & $C(13)-C(14)-C(25)$ & $119.8(4)$ \\
\hline $\mathrm{C}(1)-\mathrm{P}(1)-\mathrm{Li}(1)$ & $60.1(2)$ & $C(15)-C(14)-C(25)$ & $123.2(4)$ \\
\hline $\mathrm{C}(41)-\mathrm{P}(1)-\operatorname{Li}(1)$ & $136.9(2)$ & $\mathrm{C}(14)-\mathrm{C}(15)-\mathrm{C}(16)$ & $125.4(4)$ \\
\hline $\mathrm{C}(31)-\mathrm{P}(1)-\mathrm{Li}(1)$ & $118.3(2)$ & $\mathrm{C}(14)-\mathrm{C}(15)-\mathrm{H}(15 \mathrm{~A})$ & 117.4 \\
\hline $\mathrm{C}(11)-\mathrm{O}(1)-\mathrm{Li}(2) \# 1$ & $114.0(3)$ & $\mathrm{C}(16)-\mathrm{C}(15)-\mathrm{H}(15 \mathrm{~A})$ & 117.2 \\
\hline $\mathrm{C}(11)-\mathrm{O}(1)-\mathrm{Li}(1)$ & $88.7(3)$ & $\mathrm{C}(11)-\mathrm{C}(16)-\mathrm{C}(15)$ & $116.6(3)$ \\
\hline $\mathrm{Li}(2) \# 1-\mathrm{O}(1)-\mathrm{Li}(1)$ & $155.7(4)$ & $C(11)-C(16)-C(26)$ & $121.4(3)$ \\
\hline $\mathrm{C}(11)-\mathrm{O}(1)-\mathrm{Li}(2)$ & $165.5(3)$ & $C(15)-C(16)-C(26)$ & $122.0(3)$ \\
\hline $\mathrm{Li}(2) \# 1-\mathrm{O}(1)-\mathrm{Li}(2)$ & 79.4(4) & $\mathrm{C}(23)-\mathrm{C}(21)-\mathrm{C}(12)$ & $111.6(5)$ \\
\hline $\operatorname{Li}(1)-O(1)-\operatorname{Li}(2)$ & $79.0(3)$ & $\mathrm{C}(23)-\mathrm{C}(21)-\mathrm{C}(24)$ & $109.5(5)$ \\
\hline $\mathrm{C}(51)-\mathrm{N}(1)-\mathrm{P}(1)$ & $129.2(3)$ & $C(12)-C(21)-C(24)$ & 109.3(4) \\
\hline $\mathrm{C}(51)-\mathrm{N}(1)-\mathrm{Li}(1)$ & $133.9(4)$ & $\mathrm{C}(23)-\mathrm{C}(21)-\mathrm{C}(22)$ & 107.4(7) \\
\hline $\mathrm{P}(1)-\mathrm{N}(1)-\mathrm{Li}(1)$ & $96.5(3)$ & $C(12)-C(21)-C(22)$ & $111.2(5)$ \\
\hline $\mathrm{P}(1)-\mathrm{C}(1)-\mathrm{Li}(2)$ & $129.2(3)$ & $\mathrm{C}(24)-\mathrm{C}(21)-\mathrm{C}(22)$ & $107.6(6)$ \\
\hline $\mathrm{P}(1)-\mathrm{C}(1)-\mathrm{Li}(1)$ & $80.2(2)$ & $\mathrm{C}(21)-\mathrm{C}(22)-\mathrm{H}(22 \mathrm{~A})$ & 111.1 \\
\hline $\operatorname{Li}(2)-C(1)-\operatorname{Li}(1)$ & $66.7(3)$ & $\mathrm{C}(21)-\mathrm{C}(22)-\mathrm{H}(22 \mathrm{~B})$ & 107.0 \\
\hline $\mathrm{P}(1)-\mathrm{C}(1)-\mathrm{H}(1 \mathrm{~A})$ & 104.9 & $\mathrm{H}(22 \mathrm{~A})-\mathrm{C}(22)-\mathrm{H}(22 \mathrm{~B})$ & 109.5 \\
\hline $\mathrm{Li}(2)-\mathrm{C}(1)-\mathrm{H}(1 \mathrm{~A})$ & 92.6 & $\mathrm{C}(21)-\mathrm{C}(22)-\mathrm{H}(22 \mathrm{C})$ & 110.3 \\
\hline Li(1)-C(1)-H(1A) & 155.0 & $\mathrm{H}(22 \mathrm{~A})-\mathrm{C}(22)-\mathrm{H}(22 \mathrm{C})$ & 109.5 \\
\hline $\mathrm{P}(1)-\mathrm{C}(1)-\mathrm{H}(1 \mathrm{~B})$ & 104.1 & $\mathrm{H}(22 \mathrm{~B})-\mathrm{C}(22)-\mathrm{H}(22 \mathrm{C})$ & 109.5 \\
\hline Li(2)-C(1)-H(1B) & 113.5 & $\mathrm{C}(21)-\mathrm{C}(23)-\mathrm{H}(23 \mathrm{~A})$ & 109.4 \\
\hline Li(1)-C(1)-H(1B) & 91.1 & $\mathrm{C}(21)-\mathrm{C}(23)-\mathrm{H}(23 \mathrm{~B})$ & 109.5 \\
\hline $\mathrm{H}(1 \mathrm{~A})-\mathrm{C}(1)-\mathrm{H}(1 \mathrm{~B})$ & 110.8 & $\mathrm{H}(23 \mathrm{~A})-\mathrm{C}(23)-\mathrm{H}(23 \mathrm{~B})$ & 109.5 \\
\hline $\mathrm{O}(1)-\mathrm{C}(11)-\mathrm{C}(16)$ & $119.3(3)$ & $\mathrm{C}(21)-\mathrm{C}(23)-\mathrm{H}(23 \mathrm{C})$ & 109.4 \\
\hline $\mathrm{O}(1)-\mathrm{C}(11)-\mathrm{C}(12)$ & $122.2(3)$ & $\mathrm{H}(23 \mathrm{~A})-\mathrm{C}(23)-\mathrm{H}(23 \mathrm{C})$ & 109.4 \\
\hline$C(16)-C(11)-C(12)$ & $118.5(4)$ & $\mathrm{H}(23 \mathrm{~B})-\mathrm{C}(23)-\mathrm{H}(23 \mathrm{C})$ & 109.7 \\
\hline $\mathrm{O}(1)-\mathrm{C}(11)-\mathrm{Li}(1)$ & $54.9(3)$ & $\mathrm{C}(21)-\mathrm{C}(24)-\mathrm{H}(24 \mathrm{~A})$ & 109.0 \\
\hline$C(16)-C(11)-\operatorname{Li}(1)$ & $110.7(3)$ & $\mathrm{C}(21)-\mathrm{C}(24)-\mathrm{H}(24 \mathrm{~B})$ & 109.4 \\
\hline $\mathrm{C}(12)-\mathrm{C}(11)-\mathrm{Li}(1)$ & $102.1(4)$ & $\mathrm{H}(24 \mathrm{~A})-\mathrm{C}(24)-\mathrm{H}(24 \mathrm{~B})$ & 109.5 \\
\hline$C(13)-C(12)-C(11)$ & $120.7(4)$ & $\mathrm{C}(21)-\mathrm{C}(24)-\mathrm{H}(24 \mathrm{C})$ & 110.0 \\
\hline$C(13)-C(12)-C(21)$ & $118.1(4)$ & $\mathrm{H}(24 \mathrm{~A})-\mathrm{C}(24)-\mathrm{H}(24 \mathrm{C})$ & 109.5 \\
\hline$C(11)-C(12)-C(21)$ & $121.2(4)$ & $\mathrm{H}(24 \mathrm{~B})-\mathrm{C}(24)-\mathrm{H}(24 \mathrm{C})$ & 109.5 \\
\hline$C(14)-C(13)-C(12)$ & $121.8(4)$ & $\mathrm{C}(14)-\mathrm{C}(25)-\mathrm{H}(25 \mathrm{~A})$ & 109.5 \\
\hline $\mathrm{C}(14)-\mathrm{C}(13)-\mathrm{H}(13 \mathrm{~A})$ & 119.1 & $\mathrm{C}(14)-\mathrm{C}(25)-\mathrm{H}(25 \mathrm{~B})$ & 109.4 \\
\hline
\end{tabular}




\begin{tabular}{|c|c|c|c|}
\hline $\mathrm{H}(25 \mathrm{~A})-\mathrm{C}(25)-\mathrm{H}(25 \mathrm{~B})$ & 109.5 & $\mathrm{C}(32)-\mathrm{C}(33)-\mathrm{H}(33 \mathrm{~A})$ & 120.6 \\
\hline $\mathrm{C}(14)-\mathrm{C}(25)-\mathrm{H}(25 \mathrm{C})$ & 109.5 & $\mathrm{C}(33)-\mathrm{C}(34)-\mathrm{C}(35)$ & $119.7(5)$ \\
\hline $\mathrm{H}(25 \mathrm{~A})-\mathrm{C}(25)-\mathrm{H}(25 \mathrm{C})$ & 109.5 & $\mathrm{C}(33)-\mathrm{C}(34)-\mathrm{H}(34 \mathrm{~A})$ & 120.2 \\
\hline $\mathrm{H}(25 \mathrm{~B})-\mathrm{C}(25)-\mathrm{H}(25 \mathrm{C})$ & 109.5 & $\mathrm{C}(35)-\mathrm{C}(34)-\mathrm{H}(34 \mathrm{~A})$ & 120.2 \\
\hline$C(27)-C(26)-C(29)$ & $106.8(3)$ & $\mathrm{C}(36)-\mathrm{C}(35)-\mathrm{C}(34)$ & $122.6(5)$ \\
\hline$C(27)-C(26)-C(28)$ & $106.7(3)$ & $\mathrm{C}(36)-\mathrm{C}(35)-\mathrm{H}(35 \mathrm{~A})$ & 118.7 \\
\hline$C(29)-C(26)-C(28)$ & $108.0(3)$ & $\mathrm{C}(34)-\mathrm{C}(35)-\mathrm{H}(35 \mathrm{~A})$ & 118.8 \\
\hline$C(27)-C(26)-C(16)$ & $110.6(3)$ & $\mathrm{C}(31)-\mathrm{C}(36)-\mathrm{C}(35)$ & $117.8(5)$ \\
\hline$C(29)-C(26)-C(16)$ & $112.8(3)$ & $\mathrm{C}(31)-\mathrm{C}(36)-\mathrm{H}(36 \mathrm{~A})$ & 121.1 \\
\hline$C(28)-C(26)-C(16)$ & $111.7(3)$ & $\mathrm{C}(35)-\mathrm{C}(36)-\mathrm{H}(36 \mathrm{~A})$ & 121.0 \\
\hline $\mathrm{C}(26)-\mathrm{C}(27)-\mathrm{H}(27 \mathrm{~A})$ & 109.6 & $\mathrm{C}(42)-\mathrm{C}(41)-\mathrm{C}(46)$ & $120.5(4)$ \\
\hline $\mathrm{C}(26)-\mathrm{C}(27)-\mathrm{H}(27 \mathrm{~B})$ & 109.4 & $\mathrm{C}(42)-\mathrm{C}(41)-\mathrm{P}(1)$ & $124.2(3)$ \\
\hline $\mathrm{H}(27 \mathrm{~A})-\mathrm{C}(27)-\mathrm{H}(27 \mathrm{~B})$ & 109.4 & $\mathrm{C}(46)-\mathrm{C}(41)-\mathrm{P}(1)$ & $115.3(3)$ \\
\hline $\mathrm{C}(26)-\mathrm{C}(27)-\mathrm{H}(27 \mathrm{C})$ & 109.4 & $\mathrm{C}(43)-\mathrm{C}(42)-\mathrm{C}(41)$ & $120.8(4)$ \\
\hline $\mathrm{H}(27 \mathrm{~A})-\mathrm{C}(27)-\mathrm{H}(27 \mathrm{C})$ & 109.6 & $\mathrm{C}(43)-\mathrm{C}(42)-\mathrm{H}(42 \mathrm{~A})$ & 119.6 \\
\hline $\mathrm{H}(27 \mathrm{~B})-\mathrm{C}(27)-\mathrm{H}(27 \mathrm{C})$ & 109.4 & $\mathrm{C}(41)-\mathrm{C}(42)-\mathrm{H}(42 \mathrm{~A})$ & 119.6 \\
\hline $\mathrm{C}(26)-\mathrm{C}(28)-\mathrm{H}(28 \mathrm{~A})$ & 109.5 & $\mathrm{C}(42)-\mathrm{C}(43)-\mathrm{C}(44)$ & $118.1(4)$ \\
\hline $\mathrm{C}(26)-\mathrm{C}(28)-\mathrm{H}(28 \mathrm{~B})$ & 109.4 & $\mathrm{C}(42)-\mathrm{C}(43)-\mathrm{H}(43 \mathrm{~A})$ & 121.0 \\
\hline $\mathrm{H}(28 \mathrm{~A})-\mathrm{C}(28)-\mathrm{H}(28 \mathrm{~B})$ & 109.4 & $\mathrm{C}(44)-\mathrm{C}(43)-\mathrm{H}(43 \mathrm{~A})$ & 120.9 \\
\hline $\mathrm{C}(26)-\mathrm{C}(28)-\mathrm{H}(28 \mathrm{C})$ & 109.4 & $\mathrm{C}(43)-\mathrm{C}(44)-\mathrm{C}(45)$ & $121.8(4)$ \\
\hline $\mathrm{H}(28 \mathrm{~A})-\mathrm{C}(28)-\mathrm{H}(28 \mathrm{C})$ & 109.6 & $\mathrm{C}(43)-\mathrm{C}(44)-\mathrm{H}(44 \mathrm{~A})$ & 119.2 \\
\hline $\mathrm{H}(28 \mathrm{~B})-\mathrm{C}(28)-\mathrm{H}(28 \mathrm{C})$ & 109.5 & $\mathrm{C}(45)-\mathrm{C}(44)-\mathrm{H}(44 \mathrm{~A})$ & 119.0 \\
\hline $\mathrm{C}(26)-\mathrm{C}(29)-\mathrm{H}(29 \mathrm{~A})$ & 109.5 & $\mathrm{C}(46)-\mathrm{C}(45)-\mathrm{C}(44)$ & $119.9(4)$ \\
\hline $\mathrm{C}(26)-\mathrm{C}(29)-\mathrm{H}(29 \mathrm{~B})$ & 109.5 & $\mathrm{C}(46)-\mathrm{C}(45)-\mathrm{H}(45 \mathrm{~A})$ & 120.0 \\
\hline $\mathrm{H}(29 \mathrm{~A})-\mathrm{C}(29)-\mathrm{H}(29 \mathrm{~B})$ & 109.5 & $\mathrm{C}(44)-\mathrm{C}(45)-\mathrm{H}(45 \mathrm{~A})$ & 120.0 \\
\hline $\mathrm{C}(26)-\mathrm{C}(29)-\mathrm{H}(29 \mathrm{C})$ & 109.4 & $C(45)-C(46)-C(41)$ & $118.8(5)$ \\
\hline $\mathrm{H}(29 \mathrm{~A})-\mathrm{C}(29)-\mathrm{H}(29 \mathrm{C})$ & 109.5 & $\mathrm{C}(45)-\mathrm{C}(46)-\mathrm{H}(46 \mathrm{~A})$ & 120.4 \\
\hline $\mathrm{H}(29 \mathrm{~B})-\mathrm{C}(29)-\mathrm{H}(29 \mathrm{C})$ & 109.4 & $\mathrm{C}(41)-\mathrm{C}(46)-\mathrm{H}(46 \mathrm{~A})$ & 120.7 \\
\hline $\mathrm{C}(36)-\mathrm{C}(31)-\mathrm{C}(32)$ & 119.1(4) & $\mathrm{N}(1)-\mathrm{C}(51)-\mathrm{C}(52)$ & $123.5(4)$ \\
\hline $\mathrm{C}(36)-\mathrm{C}(31)-\mathrm{P}(1)$ & $118.2(4)$ & $\mathrm{N}(1)-\mathrm{C}(51)-\mathrm{C}(56)$ & $118.8(4)$ \\
\hline $\mathrm{C}(32)-\mathrm{C}(31)-\mathrm{P}(1)$ & $122.5(3)$ & $C(52)-C(51)-C(56)$ & $117.7(4)$ \\
\hline $\mathrm{C}(33)-\mathrm{C}(32)-\mathrm{C}(31)$ & $122.2(5)$ & $\mathrm{C}(53)-\mathrm{C}(52)-\mathrm{C}(51)$ & $119.3(4)$ \\
\hline $\mathrm{C}(33)-\mathrm{C}(32)-\mathrm{H}(32 \mathrm{~A})$ & 119.0 & $\mathrm{C}(53)-\mathrm{C}(52)-\mathrm{H}(52 \mathrm{~A})$ & 120.3 \\
\hline $\mathrm{C}(31)-\mathrm{C}(32)-\mathrm{H}(32 \mathrm{~A})$ & 118.8 & $\mathrm{C}(51)-\mathrm{C}(52)-\mathrm{H}(52 \mathrm{~A})$ & 120.4 \\
\hline $\mathrm{C}(34)-\mathrm{C}(33)-\mathrm{C}(32)$ & $118.6(6)$ & $\mathrm{C}(52)-\mathrm{C}(53)-\mathrm{C}(54)$ & $121.8(4)$ \\
\hline $\mathrm{C}(34)-\mathrm{C}(33)-\mathrm{H}(33 \mathrm{~A})$ & 120.8 & $\mathrm{C}(52)-\mathrm{C}(53)-\mathrm{H}(53 \mathrm{~A})$ & 119.1 \\
\hline
\end{tabular}




\begin{tabular}{|c|c|c|c|}
\hline $\mathrm{C}(54)-\mathrm{C}(53)-\mathrm{H}(53 \mathrm{~A})$ & 119.1 & $\mathrm{C}(1)-\operatorname{Li}(2)-\operatorname{Li}(2) \# 1$ & $152.0(6)$ \\
\hline $\mathrm{C}(55)-\mathrm{C}(54)-\mathrm{C}(53)$ & $119.7(4)$ & $\mathrm{O}(1) \# 1-\mathrm{Li}(2)-\mathrm{Li}(1)$ & $148.6(5)$ \\
\hline $\mathrm{C}(55)-\mathrm{C}(54)-\mathrm{H}(54 \mathrm{~A})$ & 120.1 & $\mathrm{O}(1)-\operatorname{Li}(2)-\operatorname{Li}(1)$ & $49.6(3)$ \\
\hline $\mathrm{C}(53)-\mathrm{C}(54)-\mathrm{H}(54 \mathrm{~A})$ & 120.1 & $\mathrm{C}(1)-\operatorname{Li}(2)-\operatorname{Li}(1)$ & $60.7(3)$ \\
\hline $\mathrm{C}(56)-\mathrm{C}(55)-\mathrm{C}(54)$ & $118.6(5)$ & $\operatorname{Li}(2) \# 1-\operatorname{Li}(2)-\operatorname{Li}(1)$ & $97.9(5)$ \\
\hline $\mathrm{C}(56)-\mathrm{C}(55)-\mathrm{H}(55 \mathrm{~A})$ & 120.7 & $\mathrm{C}(202)-\mathrm{C}(201)-\mathrm{C}(206)$ & 120.0 \\
\hline $\mathrm{C}(54)-\mathrm{C}(55)-\mathrm{H}(55 \mathrm{~A})$ & 120.7 & $\mathrm{C}(202)-\mathrm{C}(201)-\mathrm{H}(201)$ & 120.0 \\
\hline $\mathrm{C}(55)-\mathrm{C}(56)-\mathrm{C}(51)$ & $122.8(4)$ & $\mathrm{C}(206)-\mathrm{C}(201)-\mathrm{H}(201)$ & 120.0 \\
\hline $\mathrm{C}(55)-\mathrm{C}(56)-\mathrm{H}(56 \mathrm{~A})$ & 118.6 & $\mathrm{C}(203)-\mathrm{C}(202)-\mathrm{C}(201)$ & 120.0 \\
\hline $\mathrm{C}(51)-\mathrm{C}(56)-\mathrm{H}(56 \mathrm{~A})$ & 118.6 & $\mathrm{C}(203)-\mathrm{C}(202)-\mathrm{H}(202)$ & 120.0 \\
\hline $\mathrm{N}(1)-\mathrm{Li}(1)-\mathrm{O}(1)$ & $157.7(5)$ & $\mathrm{C}(201)-\mathrm{C}(202)-\mathrm{H}(202)$ & 120.0 \\
\hline $\mathrm{N}(1)-\operatorname{Li}(1)-\mathrm{C}(11)$ & $138.9(4)$ & $\mathrm{C}(202)-\mathrm{C}(203)-\mathrm{C}(204)$ & 120.0 \\
\hline $\mathrm{O}(1)-\operatorname{Li}(1)-\mathrm{C}(11)$ & $36.45(19)$ & $\mathrm{C}(202)-\mathrm{C}(203)-\mathrm{H}(203)$ & 120.0 \\
\hline $\mathrm{N}(1)-\operatorname{Li}(1)-\mathrm{C}(1)$ & $77.1(3)$ & $\mathrm{C}(204)-\mathrm{C}(203)-\mathrm{H}(203)$ & 120.0 \\
\hline $\mathrm{O}(1)-\mathrm{Li}(1)-\mathrm{C}(1)$ & $103.4(3)$ & $\mathrm{C}(205)-\mathrm{C}(204)-\mathrm{C}(203)$ & 120.0 \\
\hline $\mathrm{C}(11)-\mathrm{Li}(1)-\mathrm{C}(1)$ & $139.8(3)$ & $\mathrm{C}(205)-\mathrm{C}(204)-\mathrm{H}(204)$ & 120.0 \\
\hline$N(1)-\operatorname{Li}(1)-\operatorname{Li}(2)$ & $121.7(5)$ & $\mathrm{C}(203)-\mathrm{C}(204)-\mathrm{H}(204)$ & 120.0 \\
\hline $\mathrm{O}(1)-\operatorname{Li}(1)-\operatorname{Li}(2)$ & $51.4(3)$ & $\mathrm{C}(204)-\mathrm{C}(205)-\mathrm{C}(206)$ & 120.0 \\
\hline$C(11)-\operatorname{Li}(1)-\operatorname{Li}(2)$ & $87.6(3)$ & $\mathrm{C}(204)-\mathrm{C}(205)-\mathrm{H}(205)$ & 120.0 \\
\hline$C(1)-\operatorname{Li}(1)-\operatorname{Li}(2)$ & $52.7(3)$ & $\mathrm{C}(206)-\mathrm{C}(205)-\mathrm{H}(205)$ & 120.0 \\
\hline $\mathrm{N}(1)-\operatorname{Li}(1)-\mathrm{P}(1)$ & $38.0(2)$ & $C(205)-C(206)-C(201)$ & 120.0 \\
\hline $\mathrm{O}(1)-\operatorname{Li}(1)-\mathrm{P}(1)$ & $135.3(4)$ & $\mathrm{C}(205)-\mathrm{C}(206)-\mathrm{H}(206)$ & 120.0 \\
\hline C(11)-Li(1)-P(1) & $160.8(3)$ & $\mathrm{C}(201)-\mathrm{C}(206)-\mathrm{H}(206)$ & 120.0 \\
\hline $\mathrm{C}(1)-\operatorname{Li}(1)-\mathrm{P}(1)$ & $39.66(17)$ & $\mathrm{C}(302)-\mathrm{C}(301)-\mathrm{C}(306)$ & 120.0 \\
\hline $\operatorname{Li}(2)-\operatorname{Li}(1)-\mathrm{P}(1)$ & $85.7(3)$ & $\mathrm{C}(302)-\mathrm{C}(301)-\mathrm{H}(301)$ & 120.0 \\
\hline $\mathrm{N}(1)-\operatorname{Li}(1)-\mathrm{H}(23 \mathrm{~A})$ & 126.5 & $\mathrm{C}(306)-\mathrm{C}(301)-\mathrm{H}(301)$ & 120.0 \\
\hline $\mathrm{O}(1)-\mathrm{Li}(1)-\mathrm{H}(23 \mathrm{~A})$ & 75.7 & $\mathrm{C}(301)-\mathrm{C}(302)-\mathrm{C}(303)$ & 120.0 \\
\hline $\mathrm{C}(11)-\mathrm{Li}(1)-\mathrm{H}(23 \mathrm{~A})$ & 80.5 & $\mathrm{C}(301)-\mathrm{C}(302)-\mathrm{H}(302)$ & 120.0 \\
\hline $\mathrm{C}(1)-\mathrm{Li}(1)-\mathrm{H}(23 \mathrm{~A})$ & 89.3 & $\mathrm{C}(303)-\mathrm{C}(302)-\mathrm{H}(302)$ & 120.0 \\
\hline $\mathrm{Li}(2)-\mathrm{Li}(1)-\mathrm{H}(23 \mathrm{~A})$ & 84.5 & $\mathrm{C}(304)-\mathrm{C}(303)-\mathrm{C}(302)$ & 120.0 \\
\hline $\mathrm{P}(1)-\mathrm{Li}(1)-\mathrm{H}(23 \mathrm{~A})$ & 116.6 & $\mathrm{C}(304)-\mathrm{C}(303)-\mathrm{H}(303)$ & 120.0 \\
\hline $\mathrm{O}(1) \# 1-\mathrm{Li}(2)-\mathrm{O}(1)$ & $100.6(4)$ & $\mathrm{C}(302)-\mathrm{C}(303)-\mathrm{H}(303)$ & 120.0 \\
\hline $\mathrm{O}(1) \# 1-\mathrm{Li}(2)-\mathrm{C}(1)$ & $143.4(4)$ & $\mathrm{C}(303)-\mathrm{C}(304)-\mathrm{C}(305)$ & 120.0 \\
\hline $\mathrm{O}(1)-\mathrm{Li}(2)-\mathrm{C}(1)$ & 109.6(3) & $\mathrm{C}(303)-\mathrm{C}(304)-\mathrm{H}(304)$ & 120.0 \\
\hline $\mathrm{O}(1) \# 1-\mathrm{Li}(2)-\mathrm{Li}(2) \# 1$ & $51.7(2)$ & $\mathrm{C}(305)-\mathrm{C}(304)-\mathrm{H}(304)$ & 120.0 \\
\hline $\mathrm{O}(1)-\mathrm{Li}(2)-\mathrm{Li}(2) \# 1$ & $48.9(3)$ & $\mathrm{C}(306)-\mathrm{C}(305)-\mathrm{C}(304)$ & 120.0 \\
\hline
\end{tabular}




\begin{tabular}{|c|c|}
\hline $\mathrm{C}(306)-\mathrm{C}(305)-\mathrm{H}(305)$ & 120.0 \\
\hline $\mathrm{C}(304)-\mathrm{C}(305)-\mathrm{H}(305)$ & 120.0 \\
\hline $\mathrm{C}(305)-\mathrm{C}(306)-\mathrm{C}(301)$ & 120.0 \\
\hline $\mathrm{C}(305)-\mathrm{C}(306)-\mathrm{H}(306)$ & 120.0 \\
\hline $\mathrm{C}(301)-\mathrm{C}(306)-\mathrm{H}(306)$ & 120.0 \\
\hline $\mathrm{C}(405) \# 2-\mathrm{C}(401)-\mathrm{C}(406) \# 2$ & $111(4)$ \\
\hline $\mathrm{C}(405) \# 2-\mathrm{C}(401)-\mathrm{C}(404) \# 2$ & $91(2)$ \\
\hline $\mathrm{C}(406) \# 2-\mathrm{C}(401)-\mathrm{C}(404) \# 2$ & $143(2)$ \\
\hline $\mathrm{C}(405) \# 2-\mathrm{C}(401)-\mathrm{C}(402)$ & $113.0(16)$ \\
\hline$C(406) \# 2-C(401)-C(402)$ & $126.1(11)$ \\
\hline$C(404) \# 2-C(401)-C(402)$ & $22.4(8)$ \\
\hline$C(405) \# 2-C(401)-C(406)$ & $120(3)$ \\
\hline$C(406) \# 2-C(401)-C(406)$ & $10(2)$ \\
\hline $\mathrm{C}(404) \# 2-\mathrm{C}(401)-\mathrm{C}(406)$ & $139.7(9)$ \\
\hline$C(402)-C(401)-C(406)$ & 120.0 \\
\hline $\mathrm{C}(405) \# 2-\mathrm{C}(401)-\mathrm{C}(407)$ & $28(4)$ \\
\hline$C(406) \# 2-C(401)-C(407)$ & $98.4(8)$ \\
\hline$C(404) \# 2-C(401)-C(407)$ & $112.6(14)$ \\
\hline$C(402)-C(401)-C(407)$ & $134.2(11)$ \\
\hline$C(406)-C(401)-C(407)$ & $105.5(11)$ \\
\hline$C(403)-C(402)-C(401)$ & 120.0 \\
\hline$C(403)-C(402)-C(402) \# 2$ & $27.63(19)$ \\
\hline $\mathrm{C}(401)-\mathrm{C}(402)-\mathrm{C}(402) \# 2$ & 93.0 \\
\hline $\mathrm{C}(403)-\mathrm{C}(402)-\mathrm{H}(402)$ & 120.0 \\
\hline $\mathrm{C}(401)-\mathrm{C}(402)-\mathrm{H}(402)$ & 120.0 \\
\hline $\mathrm{C}(402) \# 2-\mathrm{C}(402)-\mathrm{H}(402)$ & 146.4 \\
\hline$C(404)-C(403)-C(402)$ & 120.0 \\
\hline $\mathrm{C}(404)-\mathrm{C}(403)-\mathrm{H}(403)$ & 120.0 \\
\hline $\mathrm{C}(402)-\mathrm{C}(403)-\mathrm{H}(403)$ & 120.0 \\
\hline$C(403)-C(404)-C(405)$ & 120.0 \\
\hline $\mathrm{C}(403)-\mathrm{C}(404)-\mathrm{H}(404)$ & 120.0 \\
\hline $\mathrm{C}(405)-\mathrm{C}(404)-\mathrm{H}(404)$ & 120.0 \\
\hline $\mathrm{C}(404)-\mathrm{C}(405)-\mathrm{C}(406)$ & 120.0 \\
\hline $\mathrm{C}(404)-\mathrm{C}(405)-\mathrm{H}(405)$ & 120.0 \\
\hline $\mathrm{C}(406)-\mathrm{C}(405)-\mathrm{H}(405)$ & 120.0 \\
\hline $\mathrm{C}(405)-\mathrm{C}(406)-\mathrm{C}(401)$ & 120.0 \\
\hline
\end{tabular}


Symmetry transformations used to generate equivalent atoms:

$\# 1-x+1 / 2,-y+1 / 2,-z+1 \quad \# 2-x, y,-z+1 / 2$ 
Table S4. Anisotropic displacement parameters $\left(\AA^{2} \times 10^{3}\right)$ for Compound 10. The anisotropic displacement factor exponent takes the form: $-2 \pi^{2}\left[h^{2} a^{* 2} U^{11}+\ldots+2 h k a^{*} b^{*} U^{12}\right]$

\begin{tabular}{|c|c|c|c|c|c|c|}
\hline & $\mathrm{U}^{11}$ & $\mathrm{U}^{22}$ & $\mathrm{U}^{33}$ & $\mathrm{U}^{23}$ & $\mathrm{U}^{13}$ & $\mathrm{U}^{12}$ \\
\hline $\mathrm{P}(1)$ & $59(1)$ & $23(1)$ & $20(1)$ & $-2(1)$ & $10(1)$ & $-14(1)$ \\
\hline $\mathrm{O}(1)$ & $55(2)$ & $24(1)$ & $15(1)$ & $1(1)$ & $-1(1)$ & $-13(1)$ \\
\hline $\mathrm{N}(1)$ & $62(2)$ & $24(2)$ & $20(1)$ & $-5(1)$ & $8(2)$ & $-11(2)$ \\
\hline $\mathrm{C}(1)$ & $71(3)$ & $28(2)$ & $24(2)$ & $-2(2)$ & $13(2)$ & $-17(2)$ \\
\hline $\mathrm{C}(11)$ & $54(3)$ & $31(2)$ & $13(2)$ & $-1(1)$ & $-2(2)$ & $-18(2)$ \\
\hline$C(12)$ & $62(3)$ & $67(3)$ & $20(2)$ & $15(2)$ & $-9(2)$ & $-38(3)$ \\
\hline$C(13)$ & $57(3)$ & $90(4)$ & $28(2)$ & $18(2)$ & $-9(2)$ & $-47(3)$ \\
\hline$C(14)$ & $50(3)$ & $60(3)$ & $23(2)$ & $6(2)$ & $-1(2)$ & $-21(2)$ \\
\hline$C(15)$ & $50(2)$ & $36(2)$ & $19(2)$ & $2(2)$ & $4(2)$ & $-7(2)$ \\
\hline$C(16)$ & $45(2)$ & $26(2)$ & $14(2)$ & $-1(1)$ & $3(2)$ & $-8(2)$ \\
\hline $\mathrm{C}(21)$ & $66(3)$ & $114(5)$ & $37(2)$ & $44(3)$ & $-22(2)$ & $-66(4)$ \\
\hline$C(22)$ & $100(6)$ & $314(15)$ & $100(6)$ & $148(8)$ & $-55(5)$ & $-138(9)$ \\
\hline$C(23)$ & 194(8) & $66(4)$ & $38(3)$ & $26(3)$ & $-35(4)$ & $-88(5)$ \\
\hline$C(24)$ & $47(3)$ & $106(5)$ & $24(2)$ & $25(2)$ & $5(2)$ & $-15(3)$ \\
\hline$C(25)$ & $50(3)$ & $87(4)$ & $38(3)$ & $15(3)$ & $-2(2)$ & $-18(3)$ \\
\hline$C(26)$ & $46(2)$ & $22(2)$ & $16(2)$ & $2(1)$ & $5(2)$ & $-5(2)$ \\
\hline $\mathrm{C}(27)$ & $50(3)$ & $32(2)$ & $24(2)$ & $10(2)$ & $9(2)$ & $-6(2)$ \\
\hline$C(28)$ & $50(2)$ & $27(2)$ & $25(2)$ & $0(1)$ & $11(2)$ & $-5(2)$ \\
\hline$C(29)$ & $47(2)$ & $22(2)$ & $22(2)$ & $1(1)$ & $10(2)$ & $-7(2)$ \\
\hline$C(31)$ & $69(3)$ & $26(2)$ & $24(2)$ & $-6(2)$ & $15(2)$ & $-21(2)$ \\
\hline$C(32)$ & $73(3)$ & $62(3)$ & $30(2)$ & $0(2)$ & $9(2)$ & $-31(3)$ \\
\hline$C(33)$ & $75(4)$ & $83(4)$ & $42(3)$ & $-8(3)$ & $14(3)$ & $-43(3)$ \\
\hline$C(34)$ & $106(5)$ & $58(3)$ & $56(3)$ & $-13(3)$ & $49(3)$ & $-44(3)$ \\
\hline$C(35)$ & $115(5)$ & $29(2)$ & $47(3)$ & $1(2)$ & $47(3)$ & $-7(3)$ \\
\hline$C(36)$ & $88(4)$ & $24(2)$ & $31(2)$ & $-4(2)$ & $30(2)$ & $-6(2)$ \\
\hline$C(41)$ & $56(3)$ & $24(2)$ & $29(2)$ & $-1(1)$ & $14(2)$ & $-9(2)$ \\
\hline$C(42)$ & $50(2)$ & $33(2)$ & $27(2)$ & $2(2)$ & $12(2)$ & $-13(2)$ \\
\hline$C(43)$ & $60(3)$ & $36(2)$ & $36(2)$ & $-1(2)$ & $19(2)$ & $-4(2)$ \\
\hline$C(44)$ & $103(4)$ & $27(2)$ & $68(3)$ & $2(2)$ & $53(3)$ & $-5(2)$ \\
\hline$C(45)$ & $129(5)$ & $26(2)$ & $104(4)$ & $-3(3)$ & $90(4)$ & $-18(3)$ \\
\hline$C(46)$ & $90(4)$ & $28(2)$ & $75(3)$ & $-7(2)$ & $54(3)$ & $-14(2)$ \\
\hline $\mathrm{C}(51)$ & $58(3)$ & $26(2)$ & $23(2)$ & $-5(1)$ & 11(2) & $-14(2)$ \\
\hline$C(52)$ & $61(3)$ & $32(2)$ & $26(2)$ & $-8(2)$ & $7(2)$ & $0(2)$ \\
\hline$C(53)$ & $70(3)$ & $45(3)$ & $26(2)$ & $-15(2)$ & $9(2)$ & $-8(2)$ \\
\hline
\end{tabular}




\begin{tabular}{lcccccc}
$\mathrm{C}(54)$ & $65(3)$ & $59(3)$ & $26(2)$ & $-9(2)$ & $1(2)$ & $-13(3)$ \\
$\mathrm{C}(55)$ & $50(3)$ & $59(3)$ & $37(2)$ & $-5(2)$ & $9(2)$ & $-9(2)$ \\
$\mathrm{C}(56)$ & $59(3)$ & $43(2)$ & $29(2)$ & $-8(2)$ & $14(2)$ & $-10(2)$ \\
$\mathrm{Li}(1)$ & $67(5)$ & $43(4)$ & $31(3)$ & $-13(3)$ & $12(3)$ & $-23(4)$ \\
$\mathrm{Li}(2)$ & $78(6)$ & $30(3)$ & $21(3)$ & $-6(3)$ & $-8(3)$ & $-9(4)$ \\
$\mathrm{C}(201)$ & $124(12)$ & $83(10)$ & $66(8)$ & $16(7)$ & $46(8)$ & $45(9)$ \\
$\mathrm{C}(202)$ & $172(15)$ & $54(7)$ & $40(6)$ & $2(5)$ & $44(8)$ & $21(8)$ \\
$\mathrm{C}(203)$ & $146(14)$ & $55(7)$ & $47(6)$ & $9(5)$ & $0(8)$ & $-9(8)$ \\
$\mathrm{C}(204)$ & $89(13)$ & $158(16)$ & $42(6)$ & $-1(8)$ & $30(7)$ & $61(12)$ \\
$\mathrm{C}(205)$ & $95(10)$ & $66(8)$ & $49(6)$ & $-12(6)$ & $9(6)$ & $0(7)$ \\
$\mathrm{C}(206)$ & $63(9)$ & $68(7)$ & $48(7)$ & $1(6)$ & $1(5)$ & $-2(7)$ \\
$\mathrm{C}(301)$ & $105(12)$ & $46(7)$ & $192(18)$ & $35(9)$ & $94(13)$ & $7(7)$ \\
$\mathrm{C}(302)$ & $76(8)$ & $69(8)$ & $106(10)$ & $-47(7)$ & $51(8)$ & $-17(7)$ \\
$\mathrm{C}(303)$ & $88(10)$ & $50(7)$ & $230(20)$ & $53(10)$ & $117(13)$ & $16(7)$ \\
$\mathrm{C}(304)$ & $162(17)$ & $65(9)$ & $290(30)$ & $-121(15)$ & $178(19)$ & $-76(11)$ \\
$\mathrm{C}(305)$ & $131(16)$ & $74(10)$ & $124(14)$ & $22(9)$ & $81(12)$ & $-23(10)$ \\
$\mathrm{C}(306)$ & $110(20)$ & $620(80)$ & $160(30)$ & $260(40)$ & $98(19)$ & $200(40)$ \\
& & & & & & \\
\hline
\end{tabular}


Table S5. Hydrogen coordinates ( $\left.\times 10^{4}\right)$ and isotropic displacement parameters $\left(\AA^{2} \times 10^{3}\right)$ for Compound $\mathbf{1 0 .}$

\begin{tabular}{|c|c|c|c|c|}
\hline & $\mathrm{x}$ & $\mathrm{y}$ & z & $\mathrm{U}(\mathrm{eq})$ \\
\hline $\mathrm{H}(1 \mathrm{~A})$ & 3202 & 371 & 4591 & $73(18)$ \\
\hline $\mathrm{H}(1 \mathrm{~B})$ & 2589 & -127 & 4610 & $56(14)$ \\
\hline $\mathrm{H}(13 \mathrm{~A})$ & 51 & 2092 & 4053 & $52(14)$ \\
\hline $\mathrm{H}(15 \mathrm{~A})$ & 676 & 3978 & 3127 & $32(11)$ \\
\hline $\mathrm{H}(22 \mathrm{~A})$ & 176 & 802 & 4558 & $130(30)$ \\
\hline $\mathrm{H}(22 \mathrm{~B})$ & 190 & 1757 & 4949 & $59(19)$ \\
\hline $\mathrm{H}(22 \mathrm{C})$ & 439 & 733 & 5243 & $190(40)$ \\
\hline $\mathrm{H}(23 \mathrm{~A})$ & 1662 & 725 & 4738 & $120(30)$ \\
\hline $\mathrm{H}(23 \mathrm{~B})$ & 1082 & 151 & 4477 & $130(30)$ \\
\hline $\mathrm{H}(23 \mathrm{C})$ & 1380 & 158 & 5157 & $130(30)$ \\
\hline $\mathrm{H}(24 \mathrm{~A})$ & 1684 & 2143 & 5433 & $38(12)$ \\
\hline $\mathrm{H}(24 \mathrm{~B})$ & 1285 & 1629 & 5754 & $110(20)$ \\
\hline $\mathrm{H}(24 \mathrm{C})$ & 1113 & 2670 & 5441 & $230(60)$ \\
\hline $\mathrm{H}(25 \mathrm{~A})$ & -614 & 2935 & 3308 & $160(40)$ \\
\hline $\mathrm{H}(25 \mathrm{~B})$ & -406 & 3179 & 2762 & $120(30)$ \\
\hline $\mathrm{H}(25 \mathrm{C})$ & -421 & 4030 & 3212 & $130(30)$ \\
\hline $\mathrm{H}(27 \mathrm{~A})$ & 1931 & 4857 & 3013 & $47(13)$ \\
\hline $\mathrm{H}(27 \mathrm{~B})$ & 1389 & 5055 & 3217 & $36(11)$ \\
\hline $\mathrm{H}(27 \mathrm{C})$ & 1377 & 4212 & 2753 & $43(12)$ \\
\hline $\mathrm{H}(28 \mathrm{~A})$ & 2480 & 3333 & 3277 & $64(16)$ \\
\hline $\mathrm{H}(28 \mathrm{~B})$ & 1927 & 2682 & 3028 & $53(14)$ \\
\hline $\mathrm{H}(28 \mathrm{C})$ & 2318 & 2517 & 3674 & $48(13)$ \\
\hline $\mathrm{H}(29 \mathrm{~A})$ & 2525 & 4663 & 3974 & $27(10)$ \\
\hline $\mathrm{H}(29 \mathrm{~B})$ & 2398 & 3883 & 4412 & $27(10)$ \\
\hline $\mathrm{H}(29 \mathrm{C})$ & 2022 & 4853 & 4241 & $42(12)$ \\
\hline $\mathrm{H}(32 \mathrm{~A})$ & 3858 & 132 & 4040 & $70(17)$ \\
\hline $\mathrm{H}(33 \mathrm{~A})$ & 4526 & 1054 & 3748 & $68(17)$ \\
\hline $\mathrm{H}(34 \mathrm{~A})$ & 4217 & 2124 & 2957 & $69(17)$ \\
\hline $\mathrm{H}(35 \mathrm{~A})$ & 3250 & 2259 & 2469 & $68(16)$ \\
\hline $\mathrm{H}(36 \mathrm{~A})$ & 2571 & 1362 & 2762 & $73(19)$ \\
\hline $\mathrm{H}(42 \mathrm{~A})$ & 3311 & -1232 & 3206 & $49(13)$ \\
\hline $\mathrm{H}(43 \mathrm{~A})$ & 3431 & -2952 & 3165 & $58(15)$ \\
\hline $\mathrm{H}(44 \mathrm{~A})$ & 2877 & -3994 & 3544 & $77(18)$ \\
\hline
\end{tabular}




\begin{tabular}{|c|c|c|c|c|}
\hline $\mathrm{H}(45 \mathrm{~A})$ & 2226 & -3353 & 3970 & $83(19)$ \\
\hline $\mathrm{H}(46 \mathrm{~A})$ & 2110 & -1638 & 4016 & $66(16)$ \\
\hline $\mathrm{H}(52 \mathrm{~A})$ & 2105 & -626 & 2531 & $36(12)$ \\
\hline $\mathrm{H}(53 \mathrm{~A})$ & 1471 & -823 & 1609 & $64(16)$ \\
\hline $\mathrm{H}(54 \mathrm{~A})$ & 573 & -88 & 1355 & $50(13)$ \\
\hline $\mathrm{H}(55 \mathrm{~A})$ & 308 & 885 & 2033 & $60(16)$ \\
\hline $\mathrm{H}(56 \mathrm{~A})$ & 935 & 1084 & 2952 & $49(13)$ \\
\hline $\mathrm{H}(201)$ & -157 & 5896 & 5436 & 104 \\
\hline $\mathrm{H}(202)$ & 445 & 7211 & 5468 & 103 \\
\hline $\mathrm{H}(203)$ & 1086 & 7146 & 4950 & 107 \\
\hline $\mathrm{H}(204)$ & 1125 & 5767 & 4400 & 113 \\
\hline $\mathrm{H}(205)$ & 523 & 4452 & 4368 & 88 \\
\hline $\mathrm{H}(206)$ & -118 & 4517 & 4886 & 76 \\
\hline $\mathrm{H}(301)$ & 914 & 5317 & 4115 & 124 \\
\hline $\mathrm{H}(302)$ & 622 & 6370 & 3330 & 94 \\
\hline $\mathrm{H}(303)$ & 655 & 8064 & 3470 & 131 \\
\hline $\mathrm{H}(304)$ & 980 & 8705 & 4394 & 177 \\
\hline $\mathrm{H}(305)$ & 1273 & 7652 & 5178 & 120 \\
\hline $\mathrm{H}(306)$ & 1240 & 5959 & 5039 & 342 \\
\hline $\mathrm{H}(402)$ & -443 & 6305 & 1791 & 78 \\
\hline $\mathrm{H}(403)$ & 129 & 5532 & 2606 & 72 \\
\hline $\mathrm{H}(404)$ & 657 & 6467 & 3376 & 81 \\
\hline $\mathrm{H}(405)$ & 612 & 8175 & 3332 & 62 \\
\hline $\mathrm{H}(406)$ & 40 & 8948 & 2517 & 86 \\
\hline $\mathrm{H}(40 \mathrm{~F})$ & -871 & 8182 & 1281 & 64 \\
\hline $\mathrm{H}(40 \mathrm{G})$ & -351 & 8868 & 1539 & 64 \\
\hline $\mathrm{H}(40 \mathrm{H})$ & -854 & 8881 & 1799 & 64 \\
\hline
\end{tabular}





d)

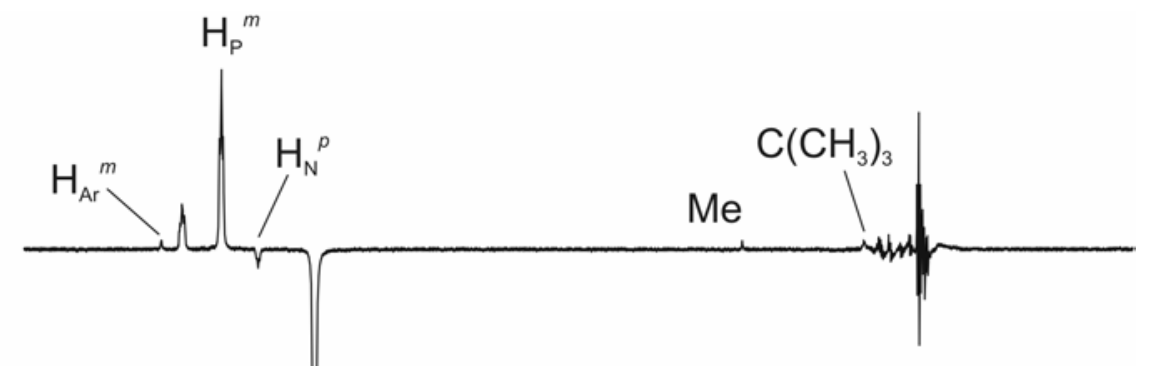

c)



b)

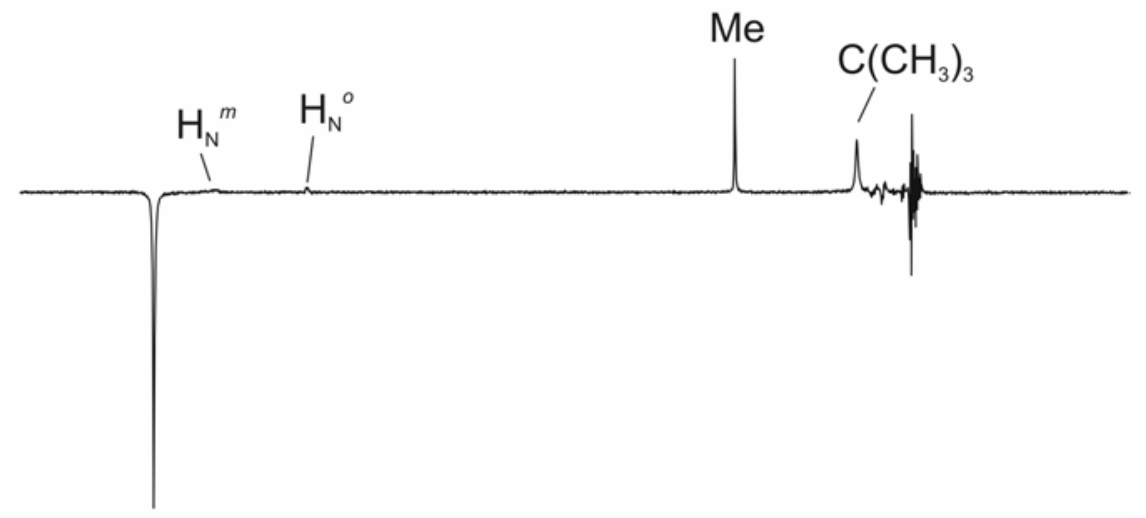

a)



Figure S3. 1D gROESY $(500.13 \mathrm{MHz})$ spectra of $\mathbf{1 0}$ at $-70{ }^{\circ} \mathrm{C}$ in toluene- $d_{8}$ corresponding to the selective excitation of (a) $\mathrm{CH}_{2} \mathrm{Li}$, (b) $\mathrm{H}_{\mathrm{Ar}}{ }^{m}$, (c) $\mathrm{H}_{\mathrm{P}}{ }^{o}$ and (d) $\mathrm{H}_{\mathrm{N}}{ }^{o}$. Negative signs denote indirect NOEs observed. 
b) ${ }^{7} \mathrm{Li},{ }^{31} \mathrm{P}\left\{{ }^{1} \mathrm{H}\right\} \mathrm{HMQC}$

a) ${ }^{7} \mathrm{Li},{ }^{31} \mathrm{P}\left\{{ }^{1} \mathrm{H}\right\} \mathrm{HMQC}$ Shift Correlation

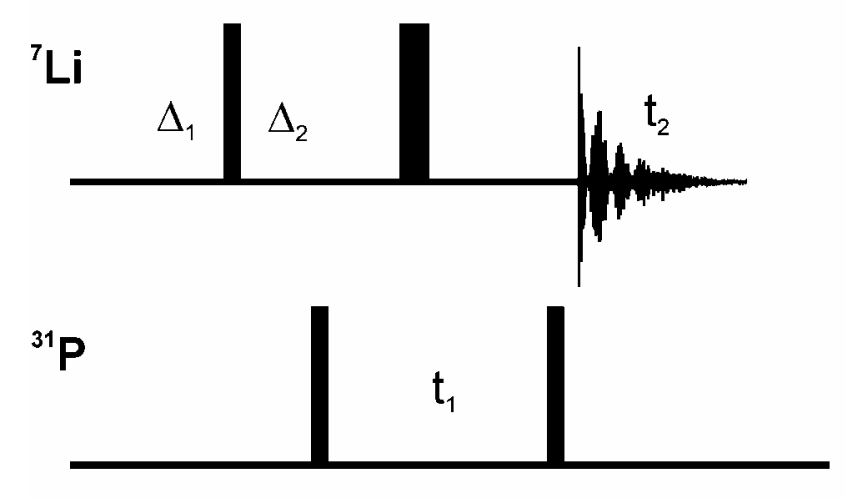

${ }^{1} \mathrm{H}$

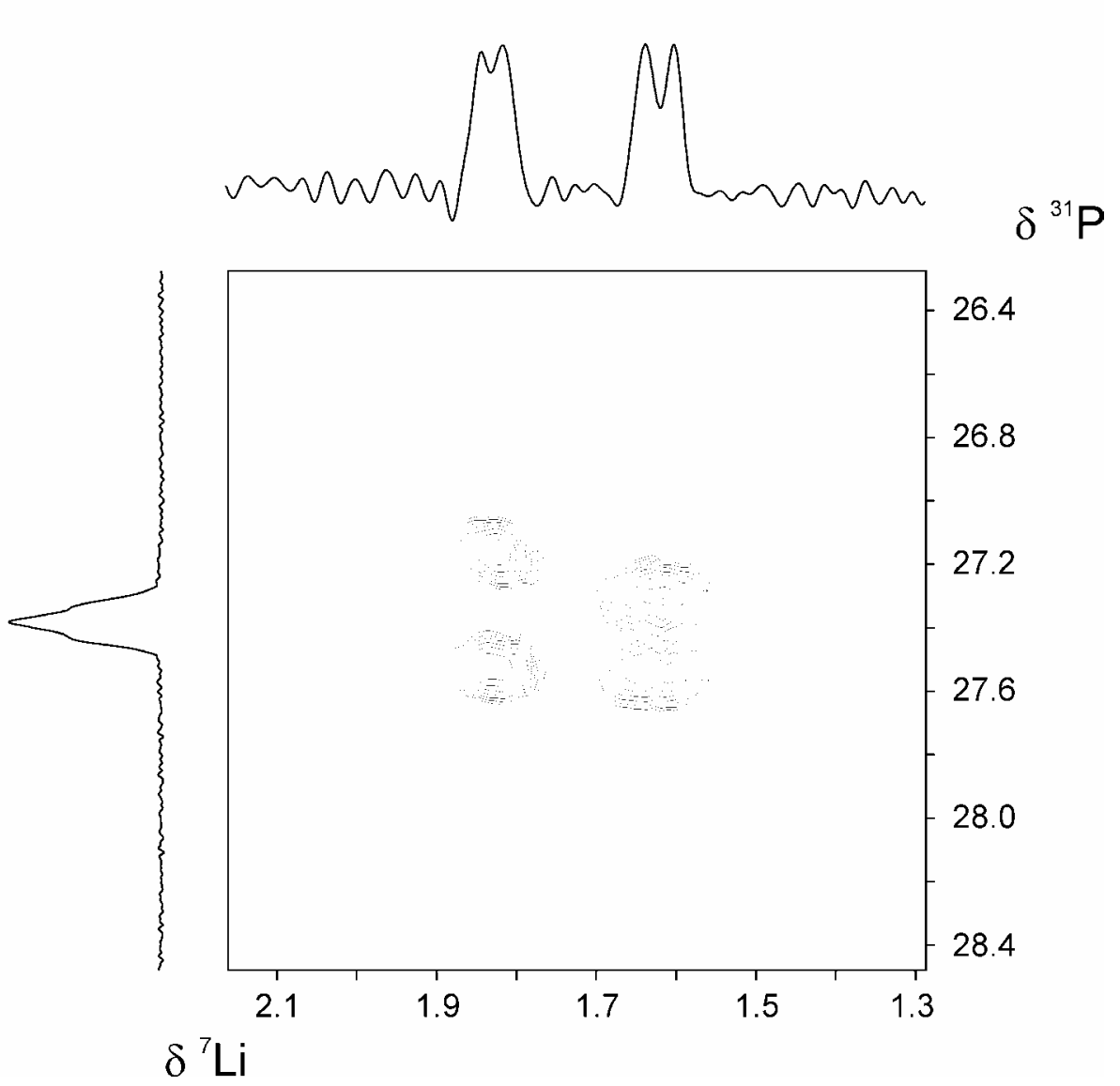

Figure S4. a) $2 \mathrm{D}{ }^{7} \mathrm{Li},{ }^{31} \mathrm{P}\left\{{ }^{1} \mathrm{H}\right\} \mathrm{HMQC}$ Shift correlation sequence, and b) $2 \mathrm{D}{ }^{7} \mathrm{Li},{ }^{31} \mathrm{P}\left\{{ }^{1} \mathrm{H}\right\}$ HMQC NMR spectrum (194.37 MHz) of complex 10 at $80{ }^{\circ} \mathrm{C}$ in toluene- $d_{8}$. Spectral width, $5200 \mathrm{~Hz}$ for ${ }^{31} \mathrm{P}$ and $1360 \mathrm{~Hz}$ for ${ }^{7} \mathrm{Li}$; 200 increments recorded; final matrix after zero filling, 2048x 128;

evolution delay of ${ }^{\mathrm{n}} J_{\mathrm{PLi}}, 16 \mathrm{~ms} ; 256$ scans per increment in $\mathrm{F} 1$; qsine multiplication of $\pi / 2$ in $\mathrm{F} 2$ and Gaussian multiplication of $\mathrm{LB}=-6, \mathrm{~GB}=0.3$ in F1 prior to transformation (magnitude mode). Repetition delay of $0.5 \mathrm{~s}$. 




Figure S5. a) ${ }^{13} \mathrm{C}\left\{{ }^{1} \mathrm{H}\right\}$ NMR spectrum $(125.75 \mathrm{MHz})$ of $\mathbf{1 0}$ measured at $-70{ }^{\circ} \mathrm{C}$ in toluene- $\left.d_{8} . \mathrm{b}\right){ }^{13} \mathrm{C}\left\{{ }^{1} \mathrm{H},{ }^{31} \mathrm{P}\right\}(125.75 \mathrm{MHz})$ expansion of the aromatic ring at the same temperature and c) expansion of the lithiated methylene region. General parameters: $22636 \mathrm{~Hz}$ of spectral width, 6144 scans accumulated, exponential multiplication of the FID $(\mathrm{LB}=2)$ was performed previous to the Fourier transformation. Unlabeled signals shown in the spectra correspond to deuterated solvent, hexane and by-products present in the $n$-BuLi hexane solution used for the deprotonation. 